Supporting Information for

\title{
Can a Single-Reference Approach Provide a Balanced Description of Ground and Excited States? A Comparison of the Completely Renormalized Equation-of-Motion Coupled-Cluster Method with Multi-Reference Quasi Degenerate Perturbation Theory Near a Conical Intersection and Along a Photodissociation Coordinate in Ammonia
}

Shikha, Nangia, ${ }^{a}$ Donald G. Truhlar, ${ }^{a}$ Michael J. McGuire, ${ }^{b}$ and Piotr Piecuch $b, c$ ${ }^{a}$ Department of Chemistry and Supercomputing Institute, University of Minnesota, Minneapolis, Minnesota 55455-0431

${ }^{b}$ Department of Chemistry, Michigan State University, East Lansing, Michigan 48824

cDepartment of Physics and Astronomy, Michigan State University, East Lansing, Michigan 48824

To be published in J. Phys. Chem. A

Date on which supporting information was prepared: October 25, 2005

S1. Adiabatic energies for scan 1 geometries.....................................

S2. Adiabatic energies for scan 2 geometries......................................

S3. Adiabatic energies for scan 3 geometries.....................................

S4. Adiabatic energies for scan 4 geometries..................................... 
Table S1. Adiabatic energies (hartrees) for the ground and first excited state of ammonia using CASSCF, MC-DQPT, CREOMCCSD(T), and EOMCCSD methods for scan 1 geometries obtained using the 6-311+G(3df,3pd) basis set.

\begin{tabular}{|c|c|c|c|c|c|c|c|c|}
\hline \multirow{2}{*}{$R_{\mathrm{A}}(\AA)$} & \multicolumn{2}{|c|}{ CASSSCF } & \multicolumn{2}{|c|}{ MC-QDPT } & \multicolumn{2}{|c|}{ CR-EOMCCSD(T) } & \multicolumn{2}{|c|}{ EOMCCSD } \\
\hline & $V_{1}$ & $V_{2}$ & $V_{1}$ & $V_{2}$ & $V_{1}$ & $V_{2}$ & $V_{1}$ & $V_{2}$ \\
\hline 0.80 & -56.194 & -56.007 & -56.397 & -56.183 & -56.411 & -56.186 & -56.404 & -56.183 \\
\hline 0.90 & -56.236 & -56.054 & -56.442 & -56.232 & -56.455 & -56.236 & -56.449 & -56.233 \\
\hline 1.00 & -56.245 & -56.067 & -56.452 & -56.249 & -56.466 & -56.253 & -56.459 & -56.249 \\
\hline 1.10 & -56.238 & -56.064 & -56.444 & -56.251 & -56.458 & -56.253 & -56.451 & -56.249 \\
\hline 1.20 & -56.225 & -56.056 & -56.429 & -56.249 & -56.441 & -56.247 & -56.433 & -56.243 \\
\hline 1.30 & -56.210 & -56.054 & -56.409 & -56.237 & -56.419 & -56.244 & -56.411 & -56.238 \\
\hline 1.40 & -56.193 & -56.061 & -56.387 & -56.236 & -56.396 & -56.245 & -56.387 & -56.237 \\
\hline 1.50 & -56.174 & -56.069 & -56.364 & -56.242 & -56.372 & -56.249 & -56.364 & -56.239 \\
\hline 1.60 & -56.156 & -56.078 & -56.343 & -56.244 & -56.350 & -56.254 & -56.341 & -56.242 \\
\hline 1.80 & -56.123 & -56.093 & -56.306 & -56.261 & -56.312 & -56.264 & -56.302 & -56.247 \\
\hline 1.90 & -56.110 & -56.099 & -56.291 & -56.273 & -56.296 & -56.268 & -56.286 & -56.248 \\
\hline 2.00 & -56.104 & -56.099 & -56.278 & -56.273 & -56.283 & -56.271 & -56.271 & -56.250 \\
\hline 2.10 & -56.107 & -56.090 & -56.275 & -56.268 & -56.274 & -56.272 & -56.259 & -56.250 \\
\hline 2.20 & -56.110 & -56.083 & -56.277 & -56.259 & -56.276 & -56.262 & -56.251 & -56.248 \\
\hline 2.30 & -56.113 & -56.077 & -56.278 & -56.252 & -56.278 & -56.255 & -56.251 & -56.240 \\
\hline 2.40 & -56.114 & -56.073 & -56.279 & -56.247 & -56.280 & -56.249 & -56.251 & -56.233 \\
\hline 2.60 & -56.117 & -56.067 & -56.280 & -56.239 & -56.282 & -56.240 & -56.250 & -56.222 \\
\hline 2.80 & -56.118 & -56.063 & -56.281 & -56.235 & -56.284 & -56.235 & -56.250 & -56.215 \\
\hline 3.00 & -56.119 & -56.061 & -56.282 & -56.232 & -56.285 & -56.233 & -56.250 & -56.211 \\
\hline 5.00 & -56.120 & -56.059 & -56.282 & -56.229 & -56.288 & -56.230 & -56.247 & -56.202 \\
\hline 7.00 & -56.120 & -56.059 & -56.282 & -56.229 & -56.289 & -56.230 & -56.247 & -56.202 \\
\hline 9.00 & -56.120 & -56.059 & -56.282 & -56.229 & -56.289 & -56.230 & -56.247 & -56.202 \\
\hline 11.00 & -56.120 & -56.059 & -56.282 & -56.229 & -56.289 & -56.230 & -56.247 & -56.202 \\
\hline
\end{tabular}


Table S2. Adiabatic energies (hartrees) for the ground and first excited state of ammonia using CASSCF, MC-DQPT, CR-

EOMCCSD(T), and EOMCCSD methods for scan 2 geometries obtained using the 6-311+G(3df,3pd) basis set.

\begin{tabular}{|c|c|c|c|c|c|c|c|c|}
\hline \multirow{2}{*}{$R_{\mathrm{A}}(\AA)$} & \multicolumn{2}{|c|}{ CASSSCF } & \multicolumn{2}{|c|}{ MC-QDPT } & \multicolumn{2}{|c|}{ CR-EOMCCSD(T) } & \multicolumn{2}{|c|}{ EOMCCSD } \\
\hline & $V_{1}$ & $V_{2}$ & $V_{1}$ & $V_{2}$ & $V_{1}$ & $V_{2}$ & $V_{1}$ & $V_{2}$ \\
\hline 0.80 & -56.194 & -56.007 & -56.398 & -56.182 & -56.411 & -56.186 & -56.404 & -56.183 \\
\hline 0.90 & -56.237 & -56.054 & -56.442 & -56.232 & -56.456 & -56.235 & -56.449 & -56.233 \\
\hline 1.00 & -56.246 & -56.067 & -56.453 & -56.248 & -56.466 & -56.252 & -56.459 & -56.249 \\
\hline 1.10 & -56.238 & -56.064 & -56.444 & -56.250 & -56.459 & -56.252 & -56.451 & -56.249 \\
\hline 1.20 & -56.225 & -56.056 & -56.429 & -56.248 & -56.441 & -56.247 & -56.434 & -56.243 \\
\hline 1.30 & -56.211 & -56.054 & -56.410 & -56.237 & -56.420 & -56.243 & -56.412 & -56.237 \\
\hline 1.40 & -56.194 & -56.060 & -56.387 & -56.235 & -56.396 & -56.244 & -56.388 & -56.236 \\
\hline 1.50 & -56.175 & -56.068 & -56.365 & -56.241 & -56.373 & -56.248 & -56.365 & -56.238 \\
\hline 1.60 & -56.157 & -56.077 & -56.344 & -56.242 & -56.351 & -56.253 & -56.342 & -56.241 \\
\hline 1.90 & -56.115 & -56.094 & -56.296 & -56.266 & -56.298 & -56.265 & -56.287 & -56.246 \\
\hline 2.00 & -56.110 & -56.093 & -56.287 & -56.265 & -56.286 & -56.267 & -56.274 & -56.246 \\
\hline 2.10 & -56.110 & -56.088 & -56.280 & -56.263 & -56.278 & -56.265 & -56.264 & -56.244 \\
\hline 2.20 & -56.112 & -56.082 & -56.279 & -56.257 & -56.276 & -56.257 & -56.258 & -56.238 \\
\hline 2.30 & -56.114 & -56.076 & -56.279 & -56.251 & -56.277 & -56.247 & -56.255 & -56.230 \\
\hline 2.60 & -56.117 & -56.066 & -56.281 & -56.239 & -56.281 & -56.237 & -56.254 & -56.211 \\
\hline 2.80 & -56.119 & -56.063 & -56.281 & -56.235 & -56.282 & -56.236 & -56.255 & -56.205 \\
\hline 3.00 & -56.119 & -56.061 & -56.282 & -56.232 & -56.283 & -56.236 & -56.256 & -56.201 \\
\hline 5.00 & -56.120 & -56.059 & -56.282 & -56.229 & -56.284 & -56.238 & -56.256 & -56.195 \\
\hline 7.00 & -56.120 & -56.059 & -56.282 & -56.229 & -56.284 & -56.238 & -56.255 & -56.195 \\
\hline 9.00 & -56.120 & -56.059 & -56.282 & -56.229 & -56.284 & -56.238 & -56.255 & -56.194 \\
\hline 11.00 & -56.120 & -56.059 & -56.282 & -56.229 & -56.284 & -56.238 & -56.255 & -56.194 \\
\hline
\end{tabular}


Table S3. Adiabatic energies (hartrees) for the ground and first excited state of ammonia using CASSCF, MC-DQPT, CR-

EOMCCSD(T), and EOMCCSD methods for scan 3 geometries obtained using the 6-311+G(3df,3pd) basis set.

\begin{tabular}{|c|c|c|c|c|c|c|c|c|}
\hline \multirow{2}{*}{$R_{\mathrm{A}}(\AA)$} & \multicolumn{2}{|c|}{ CASSSCF } & \multicolumn{2}{|c|}{ MC-QDPT } & \multicolumn{2}{|c|}{ CR-EOMCCSD(T) } & \multicolumn{2}{|c|}{ EOMCCSD } \\
\hline & $V_{1}$ & $V_{2}$ & $V_{1}$ & $V_{2}$ & $V_{1}$ & $V_{2}$ & $V_{1}$ & $V_{2}$ \\
\hline 0.80 & -56.185 & -56.000 & -56.390 & -56.177 & -56.406 & -56.181 & -56.399 & -56.178 \\
\hline 0.90 & -56.229 & -56.047 & -56.435 & -56.226 & -56.450 & -56.230 & -56.443 & -56.227 \\
\hline 1.00 & -56.238 & -56.061 & -56.446 & -56.243 & -56.460 & -56.247 & -56.453 & -56.244 \\
\hline 1.10 & -56.232 & -56.058 & -56.439 & -56.244 & -56.451 & -56.248 & -56.444 & -56.244 \\
\hline 1.20 & -56.220 & -56.051 & -56.424 & -56.244 & -56.433 & -56.244 & -56.425 & -56.239 \\
\hline 1.30 & -56.206 & -56.050 & -56.405 & -56.237 & -56.410 & -56.243 & -56.402 & -56.236 \\
\hline 1.40 & -56.189 & -56.055 & -56.384 & -56.233 & -56.386 & -56.246 & -56.378 & -56.238 \\
\hline 1.50 & -56.172 & -56.064 & -56.362 & -56.238 & -56.362 & -56.252 & -56.353 & -56.241 \\
\hline 1.60 & -56.154 & -56.072 & -56.342 & -56.241 & -56.339 & -56.258 & -56.330 & -56.245 \\
\hline 1.80 & -56.124 & -56.087 & -56.307 & -56.260 & -56.299 & -56.268 & -56.289 & -56.252 \\
\hline 1.90 & -56.112 & -56.092 & -56.292 & -56.267 & -56.283 & -56.273 & -56.272 & -56.254 \\
\hline 2.00 & -56.101 & -56.097 & -56.280 & -56.267 & -56.276 & -56.268 & -56.257 & -56.255 \\
\hline 2.10 & -56.100 & -56.093 & -56.270 & -56.269 & -56.279 & -56.256 & -56.256 & -56.244 \\
\hline 2.20 & -56.103 & -56.086 & -56.270 & -56.262 & -56.282 & -56.247 & -56.257 & -56.233 \\
\hline 2.30 & -56.106 & -56.081 & -56.271 & -56.256 & -56.284 & -56.238 & -56.257 & -56.224 \\
\hline 2.40 & -56.107 & -56.076 & -56.272 & -56.251 & -56.285 & -56.232 & -56.257 & -56.216 \\
\hline 2.60 & -56.110 & -56.071 & -56.274 & -56.244 & -56.288 & -56.223 & -56.257 & -56.205 \\
\hline 2.80 & -56.111 & -56.067 & -56.274 & -56.239 & -56.289 & -56.218 & -56.256 & -56.198 \\
\hline 3.00 & -56.112 & -56.066 & -56.275 & -56.237 & -56.290 & -56.215 & -56.255 & -56.193 \\
\hline 5.00 & -56.113 & -56.064 & -56.275 & -56.234 & -56.293 & -56.212 & -56.253 & -56.185 \\
\hline 7.00 & -56.113 & -56.064 & -56.275 & -56.234 & -56.294 & -56.212 & -56.253 & -56.184 \\
\hline 9.00 & -56.113 & -56.064 & -56.275 & -56.234 & -56.294 & -56.212 & -56.253 & -56.184 \\
\hline 11.00 & -56.113 & -56.064 & -56.275 & -56.234 & -56.294 & -56.213 & -56.253 & -56.184 \\
\hline
\end{tabular}


Table S4. Adiabatic energies (hartrees) for the ground and first excited state of ammonia using CASSCF, MC-DQPT, CR-

EOMCCSD(T), and EOMCCSD methods for scan 4 geometries obtained using the 6-311+G(3df,3pd) basis set.

\begin{tabular}{|c|c|c|c|c|c|c|c|c|}
\hline \multirow{2}{*}{$R_{\mathrm{A}}(\AA)$} & \multicolumn{2}{|c|}{ CASSSCF } & \multicolumn{2}{|c|}{ MC-QDPT } & \multicolumn{2}{|c|}{ CR-EOMCCSD(T) } & \multicolumn{2}{|c|}{ EOMCCSD } \\
\hline & $V_{1}$ & $V_{2}$ & $V_{1}$ & $V_{2}$ & $V_{1}$ & $V_{2}$ & $V_{1}$ & $V_{2}$ \\
\hline 0.80 & -56.186 & -55.999 & -56.390 & -56.176 & -56.406 & -56.180 & -56.400 & -56.178 \\
\hline 0.90 & -56.229 & -56.047 & -56.435 & -56.226 & -56.450 & -56.230 & -56.443 & -56.227 \\
\hline 1.00 & -56.239 & -56.061 & -56.446 & -56.243 & -56.460 & -56.247 & -56.453 & -56.244 \\
\hline 1.10 & -56.232 & -56.058 & -56.439 & -56.243 & -56.452 & -56.247 & -56.444 & -56.244 \\
\hline 1.20 & -56.220 & -56.051 & -56.425 & -56.246 & -56.434 & -56.243 & -56.426 & -56.239 \\
\hline 1.30 & -56.207 & -56.049 & -56.406 & -56.237 & -56.411 & -56.242 & -56.403 & -56.236 \\
\hline 1.40 & -56.190 & -56.054 & -56.384 & -56.233 & -56.387 & -56.245 & -56.379 & -56.237 \\
\hline 1.50 & -56.173 & -56.063 & -56.363 & -56.237 & -56.363 & -56.251 & -56.354 & -56.240 \\
\hline 1.60 & -56.156 & -56.071 & -56.343 & -56.240 & -56.340 & -56.256 & -56.331 & -56.244 \\
\hline 1.80 & -56.126 & -56.085 & -56.308 & -56.252 & -56.302 & -56.266 & -56.291 & -56.249 \\
\hline 1.90 & -56.115 & -56.089 & -56.295 & -56.263 & -56.287 & -56.267 & -56.276 & -56.248 \\
\hline 2.00 & -56.107 & -56.091 & -56.286 & -56.262 & -56.279 & -56.261 & -56.265 & -56.243 \\
\hline 2.10 & -56.105 & -56.089 & -56.278 & -56.261 & -56.279 & -56.248 & -56.260 & -56.232 \\
\hline 2.20 & -56.105 & -56.084 & -56.274 & -56.259 & -56.281 & -56.238 & -56.259 & -56.220 \\
\hline 2.30 & -56.107 & -56.079 & -56.273 & -56.254 & -56.283 & -56.232 & -56.259 & -56.211 \\
\hline 2.40 & -56.108 & -56.076 & -56.273 & -56.250 & -56.285 & -56.228 & -56.259 & -56.203 \\
\hline 2.60 & -56.110 & -56.070 & -56.274 & -56.243 & -56.287 & -56.223 & -56.260 & -56.192 \\
\hline 2.80 & -56.112 & -56.067 & -56.275 & -56.239 & -56.288 & -56.221 & -56.261 & -56.186 \\
\hline 3.00 & -56.112 & -56.065 & -56.275 & -56.237 & -56.288 & -56.220 & -56.262 & -56.182 \\
\hline 5.00 & -56.113 & -56.064 & -56.276 & -56.234 & -56.290 & -56.220 & -56.262 & -56.177 \\
\hline 7.00 & -56.113 & -56.064 & -56.276 & -56.234 & -56.290 & -56.220 & -56.262 & -56.177 \\
\hline 9.00 & -56.113 & -56.064 & -56.276 & -56.234 & -56.290 & -56.220 & -56.261 & -56.176 \\
\hline 11.00 & -56.113 & -56.064 & -56.276 & -56.234 & -56.290 & -56.220 & -56.261 & -56.176 \\
\hline
\end{tabular}

